tikunterricht kann scheitern. Gerade die Einsicht, dass mathematische Kompetenzen nur bedingt lehrbar sind und ihr Erwerb wesentlich von den AkteurInnen (also zunächst den Lehrenden UND Lernenden) abhängt, legt Scheitern sogar sehr nahe.

Wie wirkt sich Kompetenzorientierung im Mathematikunterricht aus? Wie äußert sich in Mathefachkonferenzen und Klassenräumen Fluch und Segen der Kompetenzorientierung? Mathematiklehrende „fluchen“ über die Kompetenzorientierung, wenn sie neben dem Unterricht mit seinen Verpflichtungen ein schulinternes und natürlich kompetenzorientiertes Mathematikcurriculum schreiben sollen. Obwohl gemeinhin Konsens besteht, dass Kompetenzorientierung eine Verbesserung des Mathematikunterrichts bedeutet, fehlt es für die Umsetzung an professioneller, fachlicher Begleitung und Unterstützung und - fast noch wichtiger - an strukturellen Maßnahmen, die Lehrende entlasten, damit diese sich mit den neuen Aufgaben vertraut machen und diese bewältigen können. Wieder einmal entsteht bei Lehrenden der Eindruck, „von höherer Stelle“ eine Reform verordnet $\mathrm{zu}$ kriegen und wieder einmal soll alles anders und besser werden, aber bitte ohne die alten Zöpfe (Verpflichtungen, Vorgaben, Bildungsinhalte usw.) zu kappen. Das ist bereits ein Fluch, der über der Umsetzung der Kompetenzorientierung liegt.

Ein Segen der Kompetenzorientierung für den Mathematikunterricht ist, dass sich der Fokus im Unterricht auf Anwendung und den Erwerb mathematischer Fertigkeiten und Expertise verschiebt, und dass Lernende bereits im Mathematikunterricht selbst Gelegenheit haben, in einem Teilgebiet kompetent $\mathrm{zu}$ werden. Wo Lernende früher darauf vertröstet wurden, dass sich die Anwendung ihres mathematischen Wissens später außerhalb der Schule finden würde („Grau ist alle Theorie und grün des Lebens goldner Baum"), fordert kompetenzorientierter Mathematikunterricht, das Leben entweder in den Unterricht zu bringen oder den Mathematikunterricht über die Schule hinaus auszudehnen und das Leben durch die mathematische Brille zu erforschen. Die damit einhergehende Differenzierung und Öffnung von Schule und Mathematikunterricht ist ein Segen - für alle Beteiligten.

Schließlich müssen sich Mathematiklehrende heute eingehender als vormals fragen, was in der Mathematikstunde, der Algebravorlesung und im Stochastikseminars tatsächlich gelehrt und was gelernt wurde. Dabei genügt es nicht, mathematisches Wissen in einen Trichter zu schütten in der Annahme, dass „wenn ich gelehrt habe, haben Schüler und Studenten auch gelernt". Kompetenzorientierung im Mathematikunterricht bedeutet eher das Gegenteil: „Ich habe erst dann gelehrt, wenn die SchülerInnen gelernt haben“. Das dürfte vor allem für Gymnasien und Universitäten eine ernstzunehmende Botschaft sein.

Es bleibt somit spannend, was die Erträge kompetenzorientierten Mathematikunterrichts sein werden. Was davon Fluch oder Segen ist, das wird spätestens fassbar, wenn die Kompetenzorientierung von der nächsten Reform abgelöst werden wird.

Prof. Dr. Brigitte Lutz-Westphal, Didaktik der Mathematik, Fachbereich Mathematik und Informatik, Freie Universität Berlin, Arnimallee 3, 14195 Berlin.brigitte.lutz-westphal@math.fu-berlin.de

\title{
Aufruf zur Benennung von Kandidatinnen und Kandidaten für die Präsidiumswahlen 2018
}

Im Auftrag des Präsidenten teile ich Ihnen mit, dass die Amtszeiten von fünf Präsidiumsmitgliedern in diesem Jahr auslaufen. Es handelt sich um folgende Positionen:

○ Präsident/Präsidentin und

- Vizepräsident/Vizepräsidentin.

Diese werden vom Präsidium für zwei Jahre gewählt.

- Schriftführer/Schriftführerin,

○ zwei weitere freie Sitze im Präsidium.

Diese werden von allen DMV-Mitgliedern für vier Jahre gewählt.

Die Positionen Präsident/in, Vizepräsident/in und Schriftführer/in beinhalten einen Sitz im Vorstand.
Nach § 2a der Wahlordnung ist das Präsidium verpflichtet, für jede frei werdende Position im Präsidium wenigstens einen Kandidaten/eine Kandidatin zu benennen. Weiterhin können andere Mitglieder Wahlvorschläge machen. Diese müssen nach $\S 2$ b der Wahlordnung von mindestens zehn Mitgliedern unterstützt werden und bis zum 30. April 2018 bei der Geschäftsstelle eingegangen sein.

Ich möchte alle Mitglieder nachdrücklich bitten, Vorschläge zu machen. Es wäre wünschenswert, mehr Kandidaten als Positionen zur Auswahl zu haben.

Prof. Dr. Daniel Grieser (Schriftführer der DMV) 